\title{
Robust compact model for bipolar oxide-based resistive switching memories
}

\author{
Marc Bocquet*, Damien Deleruyelle*, Hassen Aziza*, Christophe Muller*, \\ Jean-Michel Portal*, Thomas Cabout ${ }^{\dagger}$ and Eric Jalaguier ${ }^{\dagger}$ \\ *IM2NP, UMR CNRS 7334, Aix-Marseille Université, 38 rue Joliot Curie, F-13451 Marseille Cedex 20, France \\ Email: marc.bocquet@im2np.fr \\ ${ }^{\dagger}$ CEA-Léti, Campus MINATEC, 17 avenue des Martyrs, F-38054 Grenoble Cedex 9, France \\ Email: thomas.cabout@cea.fr
}

\begin{abstract}
Emerging non-volatile memories based on resistive switching mechanisms pull intense $R \& D$ efforts from both academia and industry. Oxide-based Resistive Random Access Memories (namely OxRAM) gather noteworthy performances, such as fast write/read speed, low power, high endurance and large integration density that outperform conventional Flash memories. To fully explore new design concepts such as distributed memory in logic or biomimetic architectures, robust OxRAM compact models must be developed and implemented into electrical simulators to assess performances at a circuit level. In this paper, we propose a physics-based compact model used in electrical simulator for bipolar OxRAM memories. After uncovering the theoretical background and the set of relevant physical parameters, this model is confronted to experimental electrical data. The excellent agreement with these data suggests that this model can be confidently implemented into circuit simulators for design purpose.
\end{abstract}

\section{INTRODUCTION}

Memory devices based on resistive switching materials are currently pointed out as promising candidates to replace conventional non-volatile memory (NVM) devices based on charge-storage beyond 2x nm technological nodes [1]. Indeed, as compared to conventional floating gate technologies, Resistive RAMs (so-called RRAM) gather fast write/read operations, low power consumption, CMOS voltage compatibility and high endurance [2]. Moreover, the resistive memory element generally consists in simple Metal/Insulator/Metal (MIM) structure. Hence, whatever the underlying physics, the resistive switching memory elements may be advantageously integrated into back-end-of-line (BEOL) enabling NVM solutions to be distributed over CMOS logic. Depending on fundamental physical mechanisms responsible for resistance switching, various RRAM technologies are now categorized by the ITRS. The Redox Memory category, covered in this study, includes Conductive Bridge RAM (CBRAM) [3] and Oxide Resistive RAM (OxRAM) [4] that exhibit voltage polarity-dependent bipolar switching. Besides, RRAM technologies referred as ThermoChemical Memories (TCM), or fuse-antifuse memories, are mostly based on materials such as nickel oxide $(\mathrm{NiO})$ that exhibit unipolar switching.

In the OxRAM memory elements addressed in this paper, a MIM structure is generally composed of two passive metallic electrodes sandwiching an active layer, usually an oxygendeficient oxide. A large number of resistive switching oxides, like $\mathrm{HfO}_{2}$ or $\mathrm{Ta}_{2} \mathrm{O}_{5}$, are reported in the literature [5], [6]. Even if OxRAM technology is still in its infancy, it is commonly accepted that the Valency Change Mechanism (VCM) occurs in specific transition metal oxides and the field-assisted motion of anions, such as oxygen ions $\mathrm{O}^{2-}$, governs the bipolar resistance switching [7].

After an initial Electroforming step (cf FIG. 1b), the memory element may be reversibly switched between a High Resistance State (FIG. 1d-HRS), and a Low Resistance State (FIG. 1c-LRS). The Electroforming stage corresponds to a voltage-induced resistance switching from an initial very high resistance state (pristine state) to a conductive state. Resistive switching in OxRAM elements corresponds to an abrupt change between HRS $\left(R_{H R S}\right)$ and LRS $\left(R_{L R S}\right)$ resistances. This resistance change is achieved by sweeping a voltage across the MIM structure: set operation corresponds to a HRS-to-LRS transition at $V_{S e t}$ while reset operation enables turning back the structure into HRS state by applying $V_{\text {Reset }}$ of opposite polarity. It has to be mentioned that the Electroforming voltage $V_{\text {Forming }}$ is generally larger than $V_{\text {Set }}$ even if several groups have recently demonstrated forming-free structures by adjusting the oxygen stoichiometry of the active layer [8].

Thanks to their low operating voltage (typically $1 \mathrm{~V}$ ), their fast read/write access times (tens of nanosecond) [2] and their advantageous integration into BEOL, OxRAM memories pave the way to new design solutions such as distributed memory in logic or biomimetic architectures. Targeting efficient design solutions, the compatibility between memory elements and logic blocks must be beforehand evaluated. Hence, a robust OxRAM compact model is required to assess and validate new concepts before fabrication. This compact model must (i) rely on realistic physical mechanisms described by a set of relevant parameters; (ii) match actual experimental data (quasistatic measurements, temperature dependence, timing...); (iii) guarantee a good predictability up to the system level; (iv) fulfill a computational-efficient implementation.

In the literature, many groups have proposed physical models for Set/Reset mechanisms [8]-[14], but their intrinsic complexity excludes any implementation into electrical simulators. In contrast, other models are fully compatible with simula- 
(a)

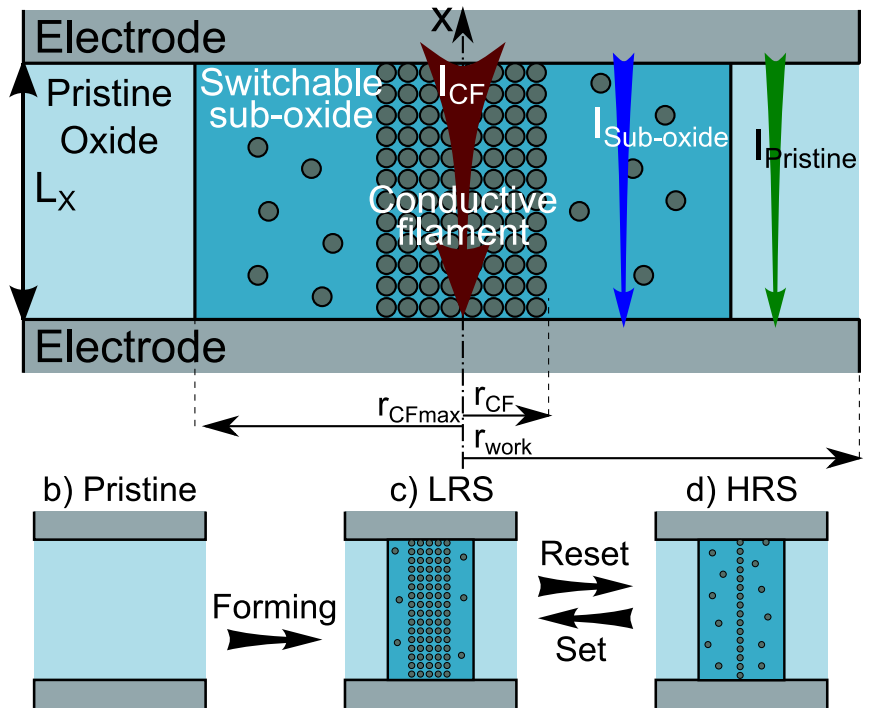

Fig. 1: Schematic representation of a) the different regions considered within the MIM structure and the different mechanism between the states of the cell: b) Pristine state, c) LRS and d) HRS.

tors (e.g. SPICE model) but do not include typical OxRAM voltage or time dependencies [15]-[17]. In this context, we propose a robust physics-based compact model for bipolar OxRAM memories that supports efficient implementation into electrical simulators. After uncovering the theoretical background and the set of relevant physical parameters, this model is confronted to experimental electrical data (DC behavior, temperature dependence, dynamical characteristics...) based on transient simulations.

\section{COMPACT MODEL FOR OXRAM MEMORY ELEMENTS}

In the literature, many works modeled the resistance switching effect by drift/diffusion of oxygen vacancies [9]-[12] Nevertheless, recent results showed that Set/Reset processes are triggered by voltage amplitude and that $V_{\text {Set }} / V_{\text {Reset }}$ are weakly dependent on temperature [18]. Given these recent insights, we propose an OxRAM model relying on local redox processes controlled by voltage polarity. In this model, the creation/destruction of a Conductive Filament (CF) is described within a switching layer by a local modification of the material (redox process). The model is based on a single master equation in which both Set and Reset operations are accounted simultaneously and controlled by the radius of the conduction pathway also called conducting filament.

To ease the implementation into electrical simulators, the model assumes an uniform $\mathrm{CF}$ radius and electric field within the oxide layer in which the temperature increase (triggered by Joule effect) may control the switching mechanisms. FIG. 1a depicts the proposed model for the switchable MIM structure. This latter is divided into three parts: a pristine oxide, switchable sub-oxide region originating from soft-breakdown events and a conductive area. In this system, the two state variables are the radius of the conductive filament $r_{C F}$ and the radius of the switchable oxide $r_{C F \max }$. The oxide thickness is named $L_{x}$ and $x=0$ corresponds to the middle of the structure.

Set and Reset operations are described by electrochemical redox reactions [13] relying on the Butler-Volmer equation [19]. In LRS, in which conduction is controlled by CF, charge transport is assumed to be ohmic accordingly to previous works reported in literature [20], [21]. On the contrary, HRS is dominated by a leakage current within the sub-oxide region. The main mechanism reported in literature is the trap-assisted conduction (Poole-Frenkel, Schottky emission, space charge limited current...), but an ohmic behavior is considered for the sake of simplicity.

\section{A. Set/Reset operation}

Set operation relies on an electrochemical reaction whose charge transfer rate can be described by the Butler-Volmer equation [19]. From this equation the electrochemical reduction rate $\tau_{\text {Red }}$ (EQ. 1) and oxidation rate $\tau_{O x}$ (EQ. 2) can be derived, here $k_{b}$ denotes the Boltzmann constant, $E_{a}$ an activation energy, $\alpha$ the charge transfer coefficient (ranging between 0 and 1) and $\tau_{\text {RedOx }}$ the nominal redox rate. The growth/destruction of the filament then results from the interplay between both redox reaction velocities through the master EQ. 3, with the CF radius $r_{C F}$ ranging from 0 to $r_{C F \max }$.

$$
\begin{gathered}
\tau_{\text {Red }}=\tau_{\text {RedOx }} \cdot e^{\frac{E_{a}-q \cdot \alpha \cdot V_{\text {Cell }}}{k_{b} \cdot T}} \\
\tau_{O x}=\tau_{\text {RedOx }} \cdot e^{\frac{E_{a}+q \cdot(1-\alpha) \cdot V_{C e l l}}{k_{b} \cdot T}} \\
\frac{d r_{C F}}{d t}=\frac{r_{C F_{\max }}-r_{C F}}{\tau_{\text {Red }}}-\frac{r_{C F}}{\tau_{O x}}
\end{gathered}
$$

where $V_{\text {Cell }}$ is the voltage applied between the top and the bottom electrodes, $q$ is the elementary charge of electron, $k_{b}$ is the Boltzmann constant, $T$ is the temperature in the structure.

\section{B. Electroforming stage}

In addition to the Set operation, Electroforming converts a highly resistive pristine oxide into a switchable sub-oxide region. After this step, standard Set/Reset operation may then occur. Due to the higher voltage bias required during Forming, with respect to Set operation, a CF is generally formed concomittantly to the sub-oxide region after Forming (FIG. 1c). The Electroforming rate $\tau_{F o r m}$ is given in EQ. 4 . The growth of the sub-oxide region is controlled by EQ. 4 , where $E_{a_{F o r m}}$ is the activation energy for Electroforming and $\tau_{F o r m 0}$ the nominal forming rate.

$$
\begin{gathered}
\tau_{\text {Form }}=\tau_{\text {Form } 0} \cdot e^{\frac{E_{a_{F o r m}}-q \cdot \alpha \cdot V_{\text {Cell }}}{k_{b} \cdot T}} \\
\frac{d r_{C F_{\max }}}{d t}=\frac{r_{w o r k}-r_{C F_{\max }}}{\tau_{\text {Form }}}
\end{gathered}
$$




\section{Temperature dependence}

As shown by Govoreanu et al. [22], temperature plays crucial role on the reaction rates. In our model, the local temperature of the filament is computed from the heat equation given in EQ. 6. Considering a cylinder-shaped filament, the temperature is given by EQ. 7. For instance, EQ. 8 gives maximum temperature reached into the $\mathrm{CF}$ at $x=0$, the middle of filament.

$$
\begin{gathered}
\sigma(x) \cdot F(x)^{2}=-K_{t h} \frac{\partial^{2} T(x)}{\partial x^{2}} \\
T(x)=T_{a m b}+\frac{V_{C e l l}^{2}}{2 \cdot L_{x}^{2} \cdot K_{t h}} \cdot\left(\frac{L_{x}^{2}}{4}-x^{2}\right) \sigma_{e q} \\
T=T_{a m b}+\frac{V_{C e l l}^{2}}{8 \cdot K_{t h}} \cdot \sigma_{e q} \\
\sigma_{e q}=\sigma_{C F} \cdot \frac{r_{C F}^{2}}{r_{w o r k}^{2}}-\sigma_{O X} \cdot \frac{r_{C F \text { max }}^{2}-r_{C F}^{2}}{r_{\text {work }}^{2}}
\end{gathered}
$$

where $\sigma(x)$ is the local electrical conductivity, $F(x)$ is the local electric field, $T_{a m b}$ is the ambient temperature and $\sigma_{C F}\left(\right.$ resp. $\left.\sigma_{O X}\right)$ the electrical conductivity of the conductive filament (resp. switchable sub-oxide) and $K_{t h}$ is the thermal conductivity. $\sigma_{e q}$ is the equivalent electrical conductivity in the work area.

Let's us mention that during Set operation, the temperature increases due to the increase of CF radius: a positive feedback loop thus occurs leading to a self-accelerated reaction. Conversely, during Reset operation, both the radius and the temperature decrease in the $\mathrm{CF}$, this feature lead to a selflimited reaction also referred as a soft Reset [23].

\section{Current through the MIM structure}

The total current flowing through the OxRAM memory element is the sum of three different contributions (EQ. 10): the first one is related to the conductive area $\left(I_{C F}\right)$; the second one that describes the conduction through the switchable suboxide $\left(I_{S u b-o x i d e}\right)$; the last contribution arises from conduction through the unswitched pristine oxide ( $\left.I_{\text {Pristine }}\right) . I_{C F}$ and $I_{\text {Sub-oxide }}$ (EQs. $11 \& 12$ respectively) are described as ohmic contributions; this assumption as already been applied efficiently for TCM [24] and has proven to be accurate without sacrificing the ease of numerical implementation. Conduction in the pristine oxide region is described by means of a tunneling current given in EQ. 13 [25].

$$
\begin{gathered}
I_{\text {Cell }}=I_{\text {Sub-oxide }}+I_{C F}+I_{\text {Pristine }} \\
I_{C F}=F \cdot \pi \cdot \sigma_{C F} \cdot r_{C F}^{2} \\
I_{S u b-\text { oxide }}=F \cdot \pi \cdot \sigma_{O X} \cdot\left(r_{C F \max }^{2}-r_{C F}^{2}\right) \\
I_{\text {Pristine }}=S_{C e l l} \cdot A \cdot F^{2} \cdot \exp \frac{-B}{F} \\
A=\frac{m_{e} \cdot q^{3}}{8 \pi \cdot h \cdot m_{e}^{o x} \cdot \phi_{b}}
\end{gathered}
$$
are provided to the model in order to take into account for the memory effect. The new filament state and the current are then computed as function of these inputs and the given time step.

where $F=\frac{V_{\text {Cell }}}{L_{x}}$ being the electric field across the active layer; $m_{e}$ and $m_{e}^{o x}$ the effective electron masses into the cathode and oxide respectively; $h$ is the Planck constant; $\phi_{b}$ the metal-oxide barrier height; $S_{C e l l}$ the section of the device.

\section{E. Numerical implementation}

The implementation of a compact model into electrical simulation tools requires a discrete resolution of a set of differential equations. If the time step is sufficiently small, $\tau_{\text {Red }}, \tau_{O x}$ and $\tau_{\text {Form }}$ be assumed constant and the discrete forms of EQs. $3 \& 5$ are given in EQs. $14 \& 15$, respectively. Solving these differential equations step by step ensures a better convergence of the simulation.

$$
\begin{gathered}
r_{C \operatorname{Fax}_{i+1}}=\left(r_{C \operatorname{mmax}_{i}}-r_{\text {work }}\right) \cdot e^{\frac{-\Delta t}{\tau_{\text {Form }}}+r_{\text {work }}} \\
r_{C F_{i+1}}=\left(r_{C F_{i}}-r_{C \max _{i}} \cdot \frac{\tau_{e q}}{\tau_{\text {Red }}}\right) \cdot e^{\frac{-\Delta t}{\tau_{e q}}}+r_{C F \max _{i}} \cdot \frac{\tau_{e q}}{\tau_{R e d}}
\end{gathered}
$$

where $\tau_{e q}=\frac{\tau_{\text {Red }} \cdot \tau_{O x}}{\tau_{\text {Red }}+\tau_{O x}}$

These equations were then implemented within an ELDO compact model following the flowchart given in FIG. 2. At each call of the OxRAM instance during a transient simulation, the previous state of the filament as well as the applied voltage 

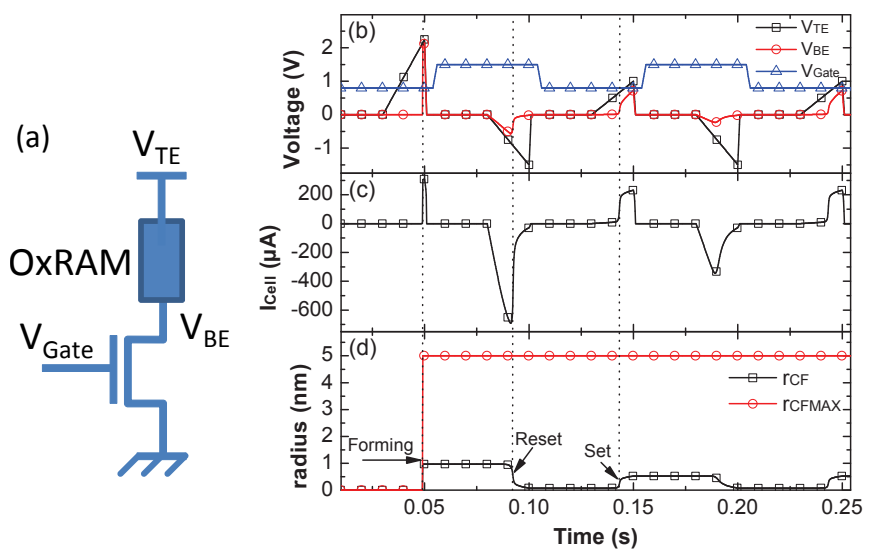

Fig. 3: Electrical simulation (ELDO) of 1T/1R OxRAM memory cell. a) Schematic of $1 \mathrm{~T} 1 \mathrm{R}$ structure. Chronogram of b) voltages applied $\left(V_{T E} \& V_{\text {Gate }}\right)$ and obtained $\left(V_{B E}\right)$ to the structure, c) current though the cell and d) radius of conductive filament.

\section{MODEL VALIDATION}

To validate the proposed theoretical approach, the model was confronted to quasi-static and dynamic experimental data extracted. First, the compact model was calibrated on recent electrical data measured on $\mathrm{HfO}_{2}$-based OxRAM devices [18]. In this study, the memory elements consisted in a $\mathrm{Ti} / \mathrm{HfO}_{2} / \mathrm{TiN}$ stack with a $5 \mathrm{~nm}$ tick hafnium oxide. The set of physical parameters used for simulations are summarized in Table I.

\section{A. DC behavior}

To fully validate the compact model and its integration into the electrical simulator, FIG. 3 gives an example of 1T/1R bipolar OxRAM memory cell simulated at a circuit level. The model used for the MOS transistor comes from Design Kit $65 \mathrm{~nm}$ STM. The simulation was performed by Eldo. This first simulation enables checking the stability of the model in a system environment, the current flowing the OxRAM being controlled by the gate voltage of the transistor. The model showed a very good stability and we have simulated 2048-bits memory array to verify the robustness and effectiveness of the model through a complex simulation.

TABLE I: Physical parameters used in bipolar OxRAM compact model

\begin{tabular}{ll}
\hline \hline$r_{\text {work }}=5 \mathrm{~nm}$ & $L_{x}=5 \mathrm{~nm}$ \\
\hline Scell $=1 \mu \mathrm{m} \times 1 \mu \mathrm{m}$ & $T_{a \mathrm{mb}}=300 \mathrm{~K}$ \\
\hline$\tau_{\text {RedOx }}=1 \times 10^{-5} \mathrm{~s}$ & $E_{a}=0.7 \mathrm{eV}$ \\
\hline$\tau_{\text {Form }}=1 \times 10^{-21} \mathrm{~s}$ & $E_{a_{F o r m}}=2.7 \mathrm{eV}$ \\
\hline$\alpha=0.7$ & $K_{t h}=2 \mathrm{~W} /(\mathrm{K} \cdot \mathrm{m})$ \\
\hline$\phi_{b}=2 \mathrm{eV}$ & $m_{e}^{o x}=0.1 \cdot \mathrm{m}_{e}$ \\
\hline$\sigma_{O x}=50 \mathrm{~m} \cdot \mathrm{S}$ & $\sigma_{C F}=5 \times 10^{6} \mathrm{~m} \cdot \mathrm{S}$ \\
\hline \hline
\end{tabular}

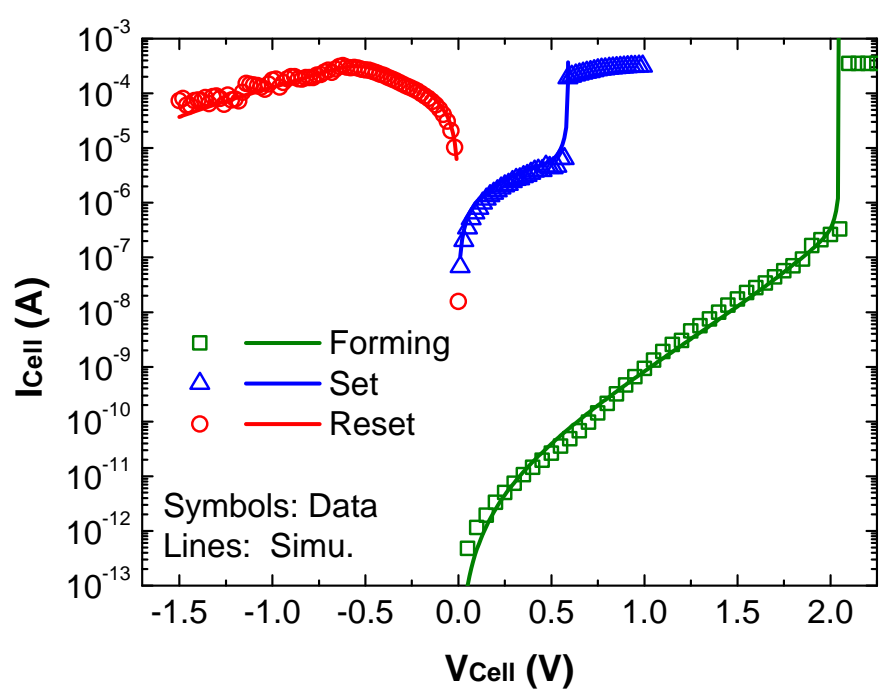

Fig. 4: Experimental $I(V)$ characteristics measured on a $\mathrm{HfO}_{2}$ based memory structures presented in [18] and corresponding simulation results from FIG. 3. The parameters of the Table I were used for simulations.

Based on this waveform, $I(V)$ characteristics were extracted. FIG. 4 shows quasi-static Electroforming, Set and Reset current-voltage $I(V)$ characteristics measured on actual $\mathrm{HfO}_{2}$-based memory elements [18]. Using the set of physical parameters given in Table I, together with the actual hafnium oxide film thickness, the present model shows an excellent agreement with the experimental data for both Set and Reset operations.

A peculiar attention must be paid to the dependence of the resistance in LRS state against the maximum current allowed during Set operations. As reported in previous works, the resistance in LRS state (noted $R_{L R S}$ ) and Reset current strongly depend on the maximum current reached during the preceding Set operation [4], [26]-[30] (referred as $I_{\text {CompSet }}$ ). This feature can be understood in terms of reduction of $\mathrm{CF}$ radius that concomitantly increases the resistance of the MIM structure [26]. FIG. 5 shows the experimental evolutions of reset current $I_{\text {reset }}$ as a function of maximum set current $I_{\text {CompSet }}$ [26]. The proposed model matches well the experimental data obtained by various authors and confirms the scalability trend of Reset current $I_{\text {reset }}$ in resistive memories. The reset current may be scaled down by limiting the maximum Set current through an integrated select device (e.g. transistor or load resistor) in series with memory element.

Another marker of OxRAM is the soft-Reset that induces a dependency between resistance in HRS $\left(R_{H R S}\right)$ and the stop voltage $\left(V_{\text {stop }}\right)$ during the preceding reset operation [23]. This feature can be understood as an incomplete destruction of the CF as shown in FIG. 1d. FIG. 6a shows that the dependence of $R_{H R S}$ versus $V_{\text {stop }}$ is well captured by the model thanks to the thermal activation of Set and Reset mechanisms. Indeed, since the filament has a uniform section, no thermal confinement occurs. There is an interplay between temperature, current 


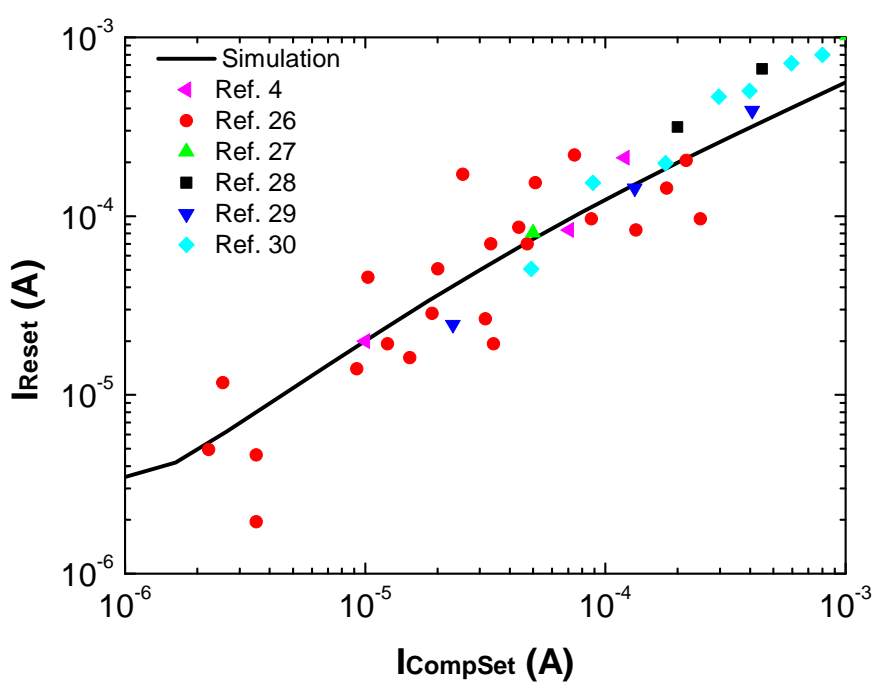

Fig. 5: Maximum current during Reset operation $\left(I_{\text {Reset }}\right)$ as a function of the maximum current during preceding Set operation $\left(I_{C o m p S e t}\right)$. Experimental data were extracted from Ref. [4], [26]-[30].

density and the $\mathrm{CF}$ radius. Since both the $\mathrm{CF}$ radius and the temperature decrease during Reset, the reaction-rate, which also depends on temperature (EQ. 2), will decrease. This feature means that the reaction-rate is self-limited leading to a soft-Reset.

\section{B. Dynamic characteristic}

Another important feature for designers is the dependence of Set and Reset switching times as a function of the applied voltage $V_{C e l l}$. FIG. $6 \mathrm{~b}$ shows switching time for Set operation (a)

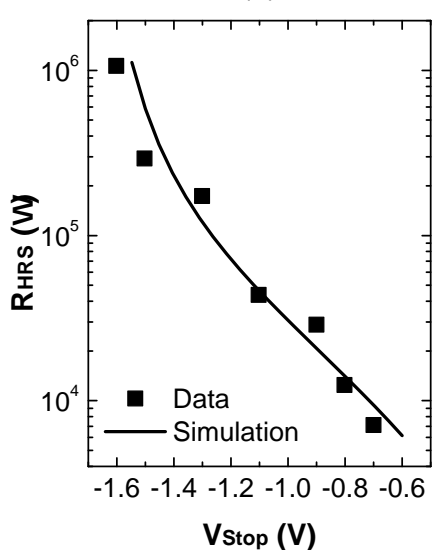

(b)

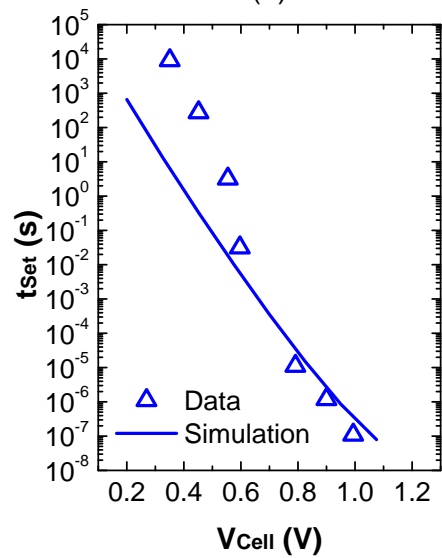

Fig. 6: a) Experimental $R_{H R S}$ as a function of stop voltage during Reset operation and b) switching time for Set operation as a function of voltage presented in [23] and corresponding simulation results.

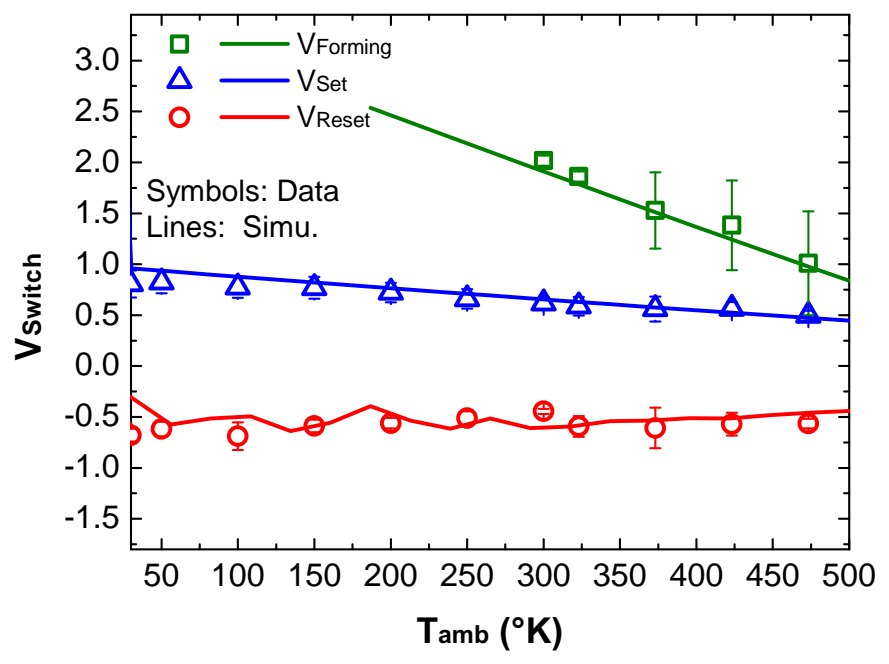

Fig. 7: Experimental switching voltage as a function of temperature in [18] and corresponding simulation results.

as a function of voltage presented in ref. [23]. The proposed model satisfactorily catches this effect using the same set of physical parameters given in Table I. It is interesting to observe that the switching time during Set operation is proportional to the reduction rate $\tau_{R e d}$. As a consequence, $\ln \left(t_{S e t}\right)$ may be expressed as a function of $V_{\text {Cell }}$ and the slope enables extracting the parameter $\alpha$ :

$$
\ln \left(t_{\text {Set }}\right)=\frac{q \cdot \alpha}{k_{b} \cdot T_{a m b}} \cdot\left(\frac{E_{a}}{q \cdot \alpha}-V_{\text {Cell }}\right)+c s t
$$

\section{Temperature dependence}

As shown in FIG. 7 and as already demonstrated in Refs. [31], [32], the Electroforming voltage $V_{\text {Forming }}$ is activated in temperature, typically $-0.005 \mathrm{~V} /{ }^{\circ} \mathrm{C}$, so that $V_{\text {Forming }}$ is almost divided by a factor 2 from room temperature (RT) to $473 \mathrm{~K}$. If RRAM switching capabilities are evaluated in temperature, one can see that both set/reset voltages exhibit less than $50 \mathrm{mV}$ variation in the investigated temperature range. Again, both of these behaviors (i.e. high thermal activation of $V_{\text {Forming }}$ and low activation of $\left.V_{\text {Set }} / V_{\text {Reset }}\right)$ are well captured by our model with the set of physical parameters given in Table I.

\section{Device-to-device variability}

Even if memory devices relying on a resistance change are attracting a lot of R\&D effort, their technological deployment is still in its infancy. Therefore, many challenging tasks such as electrical or technological variability are still to be addressed to fulfill the requirements of mass production. Let us mention that there are two relevant types of variability: device-to-device variability that characterizes the uniformity within a memory array; cycle-to-cycle variability that characterizes device stability along with cycling [33], [34]. As a consequence, there 
(a)

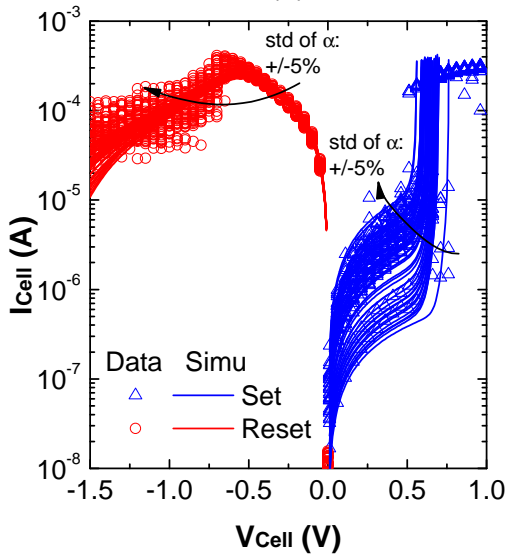

(b)

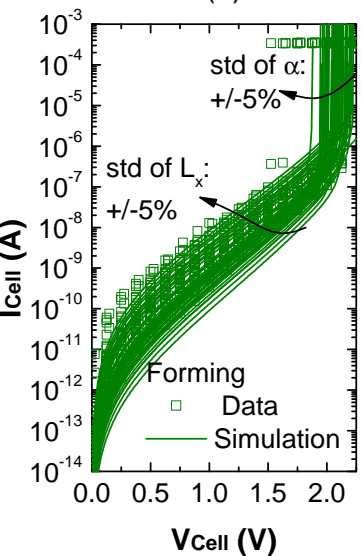

Fig. 8: Experimental $I(V)$ characteristics for Electroforming, Set and Reset measured on a large number of memory elements to reflect the device-to-device variability. Monte-Carlo simulations with a $\pm 5 \%$ standard deviation on parameters $\alpha$ and $L_{x}$ enable accounting for experimental device-to-device variability.

is an increasing demand to implement such variability in the compact model to apprehend their impact at a circuit level.

FIG. 8 depicts $I(V)$ characteristics for Electroforming, Set and Reset operations measured on a large number of memory elements coming from [18], [23]. A standard deviation of about $\pm 5 \%$ on selected parameters $\left(\alpha\right.$ and $\left.L_{x}\right)$ enables satisfactorily accounting for the device-to-device variability. This dispersion can be explained by local variations of thickness, referring to $L_{x}$, and composition, affecting $\alpha$. A modulation of $\alpha$ impacts the creation/destruction kinetics of the CF which - in turn introduces a variability in the programming voltage $\left(V_{\text {Set }} \&\right.$ $\left.V_{\text {Reset }}\right)$ and in the CF width. In the other hand, $L x$ variation impacts the current though the virgin oxide, thus the current before forming. All of these variations can be interpreted in terms of local structural or chemical variation of the oxide: crystallinity, grain boundaries, and interface roughness.

FIG. 9 shows the cumulative distributions of switching voltages (FIG. 9a) and resistances in pristine and HRS states (FIG. 9b) extracted from $I(V)$ curves measured on few tens of devices (FIG. 8). The resistance in LRS is controlled by a select transistor. Since the amount of current available during Set controls $R_{L R S}$ it is likely that $R_{L R S}$ is partially impacted by the transsitor variability which not studied here. Electrically, these variations impact Set and Reset voltages (FIG. 9a), but also the LRS (resp. HRS) resistance. Note that the parameters variation used to plot FIG. 9 simulation data are included in a distribution following a Gaussian law.

FIG. 9b plots the pristine and HRS resistance variations for the OxRAM considered in this study. The model (lines) is consistent with experimental trends (symbols). Since LRS is mostly controlled by external compliance current $\left(I_{C o m p S e t}\right)$, pristine and high resistance state are generally more affected than LRS in terms of electrical variation.

(a)

(b)

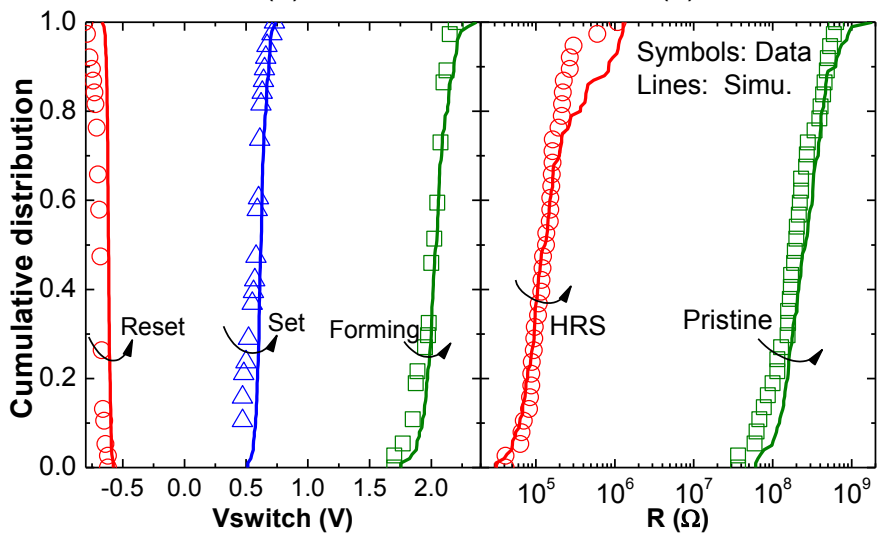

Fig. 9: a) Cumulative distributions of Electroforming, Reset and Set voltages; b) cumulative distributions of $R_{H R S}$ and $R_{\text {Pristine }}$ extracted from FIG. 8.

\section{CONCLUSION}

In conclusion, this paper deals with a physics-based compact model that is demonstrated robust for simultaneously describing Electroforming, Set and Reset operations in bipolar resistive switching memories based on $\mathrm{HfO}_{2}$ active layer. By gathering local electrochemical reactions and heat equation in a single master equation, the model enables accounting for both creation and destruction of conductive filaments. The simulation results satisfactorily match quasi-static and dynamic experimental data measured on actual resistive switching devices. Beside, the compact model may be used as a suitable tool for predicting the temperature dependence of switching parameters. Finally, the model fulfills the expectations in terms of implementation into circuit simulators and enables forecasting relevant trends required for designing innovative biomimetic architectures or for proposing novel solutions of distributed memory in logic.

\section{APPENDIX A}

\section{Set/Reset EQUATION BASED ON REDOX KINETIC}

The ButlerVolmer equation describes the electrical current on an electrode with respect to the electrode potential [19]:

$$
j=j_{0} \cdot\left[e^{\frac{\alpha \cdot z \cdot F}{R \cdot T} \cdot\left(E-E_{e q}\right)}-e^{\frac{(1-\alpha) \cdot z \cdot F}{R \cdot T} \cdot\left(E-E_{e q}\right)}\right]
$$

$j$ is the current density, $j_{o}$ the exchange current density, $E$ is the electrode potential, $E_{e q}$ is the equilibrium potential, $T$ is the temperature, $z$ is the number of electrons involved in the electrode reaction, $F$ is the Faraday constant, $R$ is the universal gas constant, $\alpha$ is the charge transfer coefficient. This expression derives from the electrochemical kinetics through a simple redox reaction, that is to say a single step mechanism:

$$
\mathrm{O}^{z+}+z \cdot e^{-\underset{r e d}{\stackrel{o x}{\leftrightarrows}} \mathrm{R}}
$$


with $\mathrm{R}$ is the reductant and $\mathrm{O}$ is the oxidant. In this case, the reaction rates for both reduction and oxidation processes may be expressed by the classical expression:

$$
\begin{gathered}
\nu_{r e d}=k_{0} e^{-\frac{\Delta r G_{0}+\alpha \cdot z \cdot F \cdot\left(E-E_{e q}\right)}{R \cdot T}} \cdot C_{O} \\
\nu_{o x}=k_{0} e^{-\frac{\Delta r G_{0}-(1-\alpha) \cdot z \cdot F \cdot\left(E-E_{e q}\right)}{R \cdot T}} \cdot C_{R} \\
\frac{d C_{A}}{d t}=\nu_{r e d}-\nu_{o x}
\end{gathered}
$$

$C_{R}$ and $C_{O}=1-C_{R}$ denotes the dimensionless concentration of the metallic and ionic species. To determine the potential of the reduced and oxidized species, the mechanism is supposed to be isotropic and the redox potential $\left(E-E_{e q}\right)$ is assumed to be equal to $-\left|V_{C e l l}\right|$. Regarding Eq.18, we assumed that electron density available during Set could not limit the reduction process. Indeed, during Set the growth of the $\mathrm{CF}$ is initiated at the cathode side. As the CF grows toward the anode, it is assumed to act as "virtual cathode" [13] allowing electrons to flow freely from the real cathode toward the reduction site.

Finally using $E_{a}[J]$ rather than $\Delta r G_{0}\left[J \cdot \mathrm{mol}^{-1}\right], \mathrm{CF}$ radius $\left(r_{C F}=r_{C F_{\max }} \times C_{R}\right)$ is calculated from the concentration of metallic species (Eq. 21) and the description of Set and Reset mechanisms enables proposing a self-consistent master equation relying on a chemical kinetics EQ. 3.

$$
\begin{gathered}
\tau_{R e d}=\tau_{R e d O x} \cdot e^{\frac{E_{a}-q \cdot \alpha \cdot V_{\text {Cell }}}{k_{b} \cdot T}} \\
\tau_{O x}=\tau_{R e d O x} \cdot e^{\frac{E_{a}+q \cdot(1-\alpha) \cdot V_{C e l l}}{k_{b} \cdot T}} \\
\frac{d r_{C F}}{d t}=\frac{r_{C F_{\max }}-r_{C F}}{\tau_{\text {Red }}}-\frac{r_{C F}}{\tau_{O x}} \\
\text { with } k_{0} e^{-\frac{\Delta r G_{0}}{R \cdot T}}=\frac{1}{\tau_{R e d O x} \cdot e^{\frac{E_{a}}{k_{b} \cdot T}}}
\end{gathered}
$$

\section{APPENDIX B}

\section{EXPRESSION ANALITIQUE}

It is interesting to note that an analytical expression of $R_{H R S}$ as a function of stop voltage during Reset operation can be expressed simply if $r_{C F_{H R S}} \neq 0$ :

$$
R_{H R S} \sim \frac{L_{x} \cdot V_{\text {Stop }}}{8 \pi K_{t h} \cdot r_{w o r k}^{2}\left(\frac{E_{a}+q(1-\alpha) V_{\text {Stop }}}{k_{b} \cdot \ln \left(\frac{t}{\tau_{\text {RedOx }}}\right)}-T_{a m b}\right)}
$$

It is interesting to observe that the switching time during the $S e t$ is directly proportional to $\tau_{R e d}$. In this way the slope of $\ln \left(t_{\text {switch }}\right)$ can be explain :

$$
\begin{gathered}
\ln \left(t_{\text {Set }}\right)=\frac{q \cdot \alpha}{k_{b} \cdot T_{a m b}} \cdot\left(\frac{E_{a}}{q \cdot \alpha}-V_{\text {Cell }}\right)+c s t \\
\ln \left(t_{\text {Forming }}\right)=\frac{q \cdot \alpha}{k_{b} \cdot T_{a m b}} \cdot\left(\frac{E_{a_{F o r m}}}{q \cdot \alpha}-V_{\text {Cell }}\right)+c s t
\end{gathered}
$$

The analytic expressions of $V_{S e t}$ and $V_{F o r m}$ can be obtained from the expressions of the kinetic reaction Set (EQ. 1) and Electroforming (EQ. 4):

$$
\begin{aligned}
V_{S e t} & \sim \frac{E_{a}}{q \cdot \alpha}-\frac{k_{b} \cdot T_{a m b}}{q \cdot \alpha} \cdot \ln \left(\frac{E_{a}}{q \cdot \alpha \cdot \beta} \cdot \frac{1}{\tau_{\text {RedOx }}}\right) \\
V_{\text {Form }} & \sim \frac{E_{a_{F o r m}}}{q \cdot \alpha}-\frac{k_{b} \cdot T_{a m b}}{q \cdot \alpha} \cdot \ln \left(\frac{E_{a_{F o r m}}}{q \cdot \alpha \cdot \beta} \cdot \frac{1}{\tau_{F o r m}}\right)
\end{aligned}
$$

These expressions can help to extract $E_{a}, E_{a_{F o r m}}, \tau_{R e d O x}$ and $\tau_{\text {Form }}$.

\section{REFERENCES}

[1] S. Hong, "Memory technology trend and future challenges," in Proc. IEEE International Electron Devices Meeting, San Francisco, CA, USA, 2010, pp. 292-295.

[2] B. Govoreanu, G. S. Kar, Y.-Y. Chen, V. Paraschiv, S. Kubicek, A. Fantini, I. P. Radu, L. Goux, S. Clima, R. Degraeve, N. Jossart, O. Richard, T. Vandeweyer, K. Seo, P. Hendrickx, G. Pourtois, H. Bender, L. Altimime, D. J. Wouters, J. A. Kittl, and M. Jurczak, "10x10nm ${ }^{2} \mathrm{Hf} / \mathrm{HfO}_{x}$ crossbar resistive RAM with excellent performance, reliability and low-energy operation," in Proc. IEEE International Electron Devices Meeting, Washington, DC, USA, 2011, pp. 729-732.

[3] M. Kund, G. Beitel, C.-U. Pinnow, T. Rohr, J. Schumann, R. Symanczyk, K.-D. Ufert, and G. Muller, "Conductive bridging RAM (CBRAM): an emerging non-volatile memory technology scalable to sub 20nm," in Proc. IEEE International Electron Devices Meeting, Washington, DC, USA, 2005, pp. 754-757.

[4] H. Y. Lee, P. S. Chen, T. Y. Wu, Y. S. Chen, C. C. Wang, P. J. Tzeng, C. H. Lin, F. Chen, C. H. Lien, and M.-J. Tsai, "Low power and high speed bipolar switching with a thin reactive Ti buffer layer in robust HfO2 based RRAM," in Proc. IEEE International Electron Devices Meeting, San Francisco, CA, USA, 2008, pp. 1-4.

[5] R. Waser and M. Aono, "Nanoionics-based resistive switching memories." Nature Materials, vol. 6, no. 11, pp. 833-40, Nov. 2007.

[6] W. G. Kim, M. G. Sung, S. J. Kim, J. Y. Kim, J. W. Moon, S. J. Yoon, J. N. Kim, B. G. Gyun, T. W. Kim, C. H. Kim, J. Y. Byun, W. Kim, T. O. Youn, J. H. Yoo, J. W. Oh, H. J. Kim, M. S. Joo, J. S. Roh, and S. K. Park, "Dependence of the switching characteristics of resistance random access memory on the type of transition metal oxide," in Proc. IEEE European Solid State Device Research Conference, Sep. 2010, pp. 400-403.

[7] H.-S. Ahn, S. Han, and C. S. Hwang, "Pairing of cation vacancies and gap-state creation in $\mathrm{TiO}_{2}$ and $\mathrm{HfO}_{2}$," Applied Physics Letters, vol. 90, no. 25, pp. 252908 (1-3), 2007.

[8] F. Nardi, S. Larentis, S. Balatti, D. C. Gilmer, and D. Ielmini, "Resistive switching by voltage-driven ion migration in bipolar RRAM part I : experimental study," IEEE Transactions on Electron Devices, vol. 59, no. 9, pp. 2461-2467, 2012.

[9] B. Gao, S. Yu, N. Xu, L. F. Liu, B. Sun, X. Y. Liu, R. Q. Han, J. F. Kang, B. Yu, and Y. Y. Wang, "Oxide-based RRAM switching mechanism: a new ion-transport-recombination model," in Proc. IEEE International Electron Devices Meeting, San Francisco, CA, USA, 2008, pp. 1-4.

[10] S. Larentis, F. Nardi, S. Balatti, D. C. Gilmer, and D. Ielmini, "Resistive switching by voltage-driven ion migration in bipolar RRAM part II : modeling," IEEE Transactions on Electron Devices, vol. 59, no. 9, pp. 2468-2475, Sep. 2012.

[11] D. C. Gilmer, G. Bersuker, S. Koveshnikov, M. Jo, A. Kalantarian, B. Butcher, R. Geer, Y. Nishi, P. D. Kirsch, and R. Jammy, "Asymmetry, vacancy engineering and mechanism for bipolar RRAM," in Proc. IEEE International Memory Workshop, May 2012, pp. 1-4.

[12] L. Vandelli, A. Padovani, L. Larcher, G. Bersuker, D. Gilmer, and P. Pavan, "Modeling of forming operation in $\mathrm{HfO}_{2}$-based resistive switching memories," in Proc. IEEE International Memory Workshop, 2011, pp. 1-4. 
[13] R. Waser, R. Dittmann, G. Staikov, and K. Szot, "Redox-based resistive switching memories nanoionic mechanisms, prospects, and challenges," Advanced Materials, vol. 21, no. 25-26, pp. 2632-2663, 2009.

[14] R. Degraeve, P. Roussel, L. Goux, D. Wouters, J. Kittl, L. Altimime, M. Jurczak, and G. Groeseneken, "Generic learning of TDDB applied to RRAM for improved understanding of conduction and switching mechanism through multiple filaments," in Proc. IEEE International Electron Devices Meeting, vol. 1, no. 1.2, 2010, pp. 1-30.

[15] Y. Lu, B. Gao, Y. Fu, B. Chen, L. Liu, X. Liu, and J. Kang, “A simplified model for resistive switching of oxide-based resistive random access memory devices," IEEE Electron Device Letters, vol. 33, no. 3, pp. 306-308, Mar. 2012.

[16] P. Sheridan, K.-H. Kim, S. Gaba, T. Chang, L. Chen, and W. Lu, "Device and SPICE modeling of RRAM devices." Nanoscale, vol. 3, no. 9, pp. 3833-40, Sep. 2011.

[17] J. Noh, M. Jo, C. Y. Kang, D. Gilmer, P. Kirsch, J. C. Lee, and B. H. Lee, "Development of a semiempirical compact model for DC/AC cell operation of $\mathrm{HfO}_{x}$-based ReRAMs," IEEE Electron Device Letters, vol. 34, no. 9, pp. 1133-1135, Sep. 2013

[18] T. Cabout, L. Perniola, V. Jousseaume, H. Grampeix, J. F. Nodin, A. Toffoli, M. Guillermet, E. Jalaguier, E. Vianello, G. Molas, G. Reimbold, B. D. Salvo, P. Tamburini, V. Amendola, R. Emilia, and R. J. Monnet, "Temperature impact (up to $200^{\circ} \mathrm{C}$ ) on performance and reliability of $\mathrm{HfO}_{2}$-based RRAMs," in Proc. IEEE International Memory Workshop, no. V, 2013, pp. 4-7.

[19] A. J. Bard and L. R. Faulkner, Electrochemical methods: fundamentals and applications, J. Wiley and S. Inc., Eds., New York, 2001.

[20] H. Akinaga and H. Shima, "Resistive Random Access Memory (ReRAM) based on metal oxides," Proc. IEEE, vol. 98, no. 12, pp 2237-2251, Dec. 2010

[21] H.-S. P. Wong, H.-Y. Lee, S. Yu, Y.-S. Chen, Y. Wu, P.-S. Chen, B. Lee, F. T. Chen, and M.-J. Tsai, "Metal-oxide RRAM," Proc. IEEE, vol. 100, no. 6, pp. 1951-1970, Jun. 2012.

[22] B. Govoreanu, S. Clima, I. P. Radu, Y.-Y. Chen, D. J. Wouters, and M. Jurczak, "Complementary role of field and temperature in triggering $\mathrm{ON} / \mathrm{OFF}$ switching mechanisms in $\mathrm{Hf} / \mathrm{Hf}_{2}$ resistive RAM cells," IEEE Transactions on Electron Devices, vol. 60, no. 8, pp. 1-8, 2013.

[23] T. Diokh, E. Le Roux, S. Jeannot, M. Gros-Jean, P. Candelier, J. Nodin, V. Jousseaume, L. Perniola, H. Grampeix, T. Cabout, E. Jalaguier, M. Guillermet, and B. De Salvo, "Investigation of the impact of the oxide thickness and RESET conditions on disturb in $\mathrm{HfO}_{2}$-RRAM integrated in a 65nm CMOS technology," in Proc. IEEE International Reliability Physics Symposium, 2013, pp. 3-6.

[24] U. Russo, D. Ielmini, C. Cagli, and A. L. Lacaita, "Filament Conduction and Reset Mechanism Memory ( RRAM ) Devices," IEEE Transactions on Electron Devices, vol. 56, no. 2, pp. 186-192, 2009.

[25] G. Pananakakis, G. Ghibaudo, R. Kies, and C. Papadas, "Temperature dependence of the FowlerNordheim current in metal-oxide-degenerate semiconductor structures," Journal of Applied Physics, vol. 78, no. 4, p. $2635,1995$.

[26] F. Nardi, D. Ielmini, C. Cagli, S. Spiga, M. Fanciulli, L. Goux, and D. J. Wouters, "Control of filament size and reduction of reset current below $10 \mu \mathrm{A}$ in $\mathrm{NiO}$ resistance switching memories," Solid-State Electronics, vol. 58, no. 1, pp. 42-47, Apr. 2011.

[27] S. Seo, M. J. Lee, D. H. Seo, E. J. Jeoung, D.-S. Suh, Y. S. Joung, I. K. Yoo, I. R. Hwang, S. H. Kim, I. S. Byun, J.-S. Kim, J. S. Choi, and B. H. Park, "Reproducible resistance switching in polycrystalline $\mathrm{NiO}$ films," Applied Physics Letters, vol. 85, no. 23, pp. 5655-5657, 2004.

[28] K. Kinoshita, K. Tsunoda, Y. Sato, H. Noshiro, S. Yagaki, M. Aoki, and Y. Sugiyama, "Reduction in the reset current in a resistive random access memory consisting of $\mathrm{NiOx}$ brought about by reducing a parasitic capacitance," Applied Physics Letters, vol. 93, no. 3, pp. 33506 (1-3), 2008

[29] T.-N. Fang, S. Kaza, S. Haddad, A. Chen, Y.-C. Wu, Z. Lan, S. Avanzino, D. Liao, C. Gopalan, S. Choi, S. Mahdavi, M. Buynoski, Y. Lin, C. Marrian, C. Bill, M. VanBuskirk, and M. Taguchi, "Erase mechanism for copper oxide resistive switching memory cells with nickel electrode," in Proc. IEEE International Electron Devices Meeting, San Francisco, CA, USA, 2006, pp. 1-4

[30] K. Tsunoda, K. Kinoshita, H. Noshiro, Y. Yamazaki, T. Iizuka, Y. Ito, A. Takahashi, A. Okano, Y. Sato, T. Fukano, M. Aoki, Y. Sugiyama, and T. Jizuka, "Low power and high speed switching of Ti-doped NiO ReRAM under the unipolar voltage source of less than 3V," in Proc. IEEE International Electron Devices Meeting, Washington, DC, USA 2007, pp. 767-770.

[31] B. Butcher, G. Bersuker, D. C. Gilmer, and A. Kalantarian, "Hot forming to improve memory window and uniformity of low-power $\mathrm{HfO}_{x}$-based RRAMs," in Proc. IEEE International Memory Workshop, no. V, 2012, pp. 49-52.

[32] L. Vandelli, A. Padovani, L. Larcher, G. Broglia, G. Ori, M. Montorsi, G. Bersuker, and P. Pavan, "Comprehensive physical modeling of forming and switching operations in HfO 2 RRAM devices," in Proc. IEEE International Electron Devices Meeting, no. 10, 2011, pp. 421424.

[33] A. Chen and M.-R. Lin, "Variability of resistive switching memories and its impact on crossbar array performance," in Proc. IEEE International Reliability Physics Symposium, Apr. 2011, pp. MY.7.1-MY.7.4.

[34] T. Cabout, J. Buckley, C. Cagli, V. Jousseaume, J.-F. Nodin, B. De Salvo, M. Bocquet, and C. Muller, "Resistance switching variability in $\mathrm{HfO}_{2}$-based memory structures with different electrodes," Thin Solid Films, vol. 533, pp. 19-23, 2013. 